\title{
p53-Cells’ Inbuilt Mechanism to Inhibit Cancer through Apoptosis
}

\author{
Rashmi Kumariya ${ }^{1 *}$, Amit K. Baruii\# and Smita Singh ${ }^{2 \#}$
}

${ }^{1}$ Animal Biochemistry, Division National Dairy Research Institute, Karnal, Haryana, India

${ }^{2}$ Dairy Chemistry, Division National Dairy Research Institute, Karnal, Haryana, India

\#These athurs are contributed equally

\begin{abstract}
Cancer cells continue to grow and divide, without undergoing apoptosis. p53, a tumor suppressor protein, serves as a checkpoint in prevention of cancer by inducing the abnormal cells to commit apoptosis. p53 can induce both extrinsic and intrinsic pathways of apoptosis and can also induce apoptosis by directly acting on mitochondria. Regulation of $\mathrm{p} 53$ concentration in cell is very critical and is regulated by Mdm2 which triggers degradation through ubiquitinylation. Keeping in view, the role of p53 in preventing cancer, many effective strategies can be developed to combat cancer by enhancing either function or stability of p53.
\end{abstract}

Keywords: p53; Cancer; Apoptosis; Tumor

\section{Introduction}

Cancer is a class of diseases characterized by uncontrolled growth of cells, which never die. Normal cells in the body follow an orderly path of growth, division, and death through apoptosis. Unlike regular cells, cancer cells do not experience apoptosis, which is the process of programmed cell death (PCD) that occurs in multicellular organisms, which involves a series of biochemical events that lead to a variety of morphological changes, including outward bulging of cell membrane, loss of membrane asymmetry and attachment, cell shrinkage, nuclear fragmentation, chromatin condensation, and chromosomal DNA fragmentation. So the cancer cells instead of undergoing apoptosis continue to grow and divide which leads to a mass of abnormal cells known as tumor that grows uncontrollably.

Apoptosis which plays a key role in inhibiting carcinogenesis is controlled by diverse range of cell signals either extracellularly or intracellularly. Extracellular signals must either cross the plasma membrane or transduce to affect a response. So, apoptosis can proceed through two pathways: extrinsic pathway and intrinsic pathway. p53 is a tumor suppressor protein which can activate both extrinsic and intrinsic pathways of apoptosis [1], leading to the activation of the aspartate-specific cysteine proteases (caspases) that mediate apoptosis and serves as a major barrier to carcinogenesis [2]. p53 is also known as protein 53 or tumor protein 53, encoded by the TP53 gene [3]. There are many isoforms of $\mathrm{p} 53$ known and the interactions between members of the p53 family and their isoforms have a profound effect on tumorigenesis and anticancer drug response [4]. It is known to regulate the cell cycle in multicellular organisms and thus functions as a tumor suppressor that is involved in preventing cancer through several mechanisms like, growth arrest, DNA repair and apoptosis. The growth arrest stops the progression of cell cycle, preventing replication of damaged DNA. During the growth arrest, p53 may activate the transcription of proteins involved in DNA repair. If the DNA damage proves to be irreparable, p53 can initiate apoptosis, which is the "last resort" to avoid proliferation of cells containing abnormal DNA $[5,6]$. Mutation in p53 leads to unrestrained cell cycle and reproduces the damaged DNA, leading to uncontrolled cell proliferation and cancer tumors [7]. Inactivation of p53 is responsible for majority of cancers as it is no longer functions as a tumor suppressor but also exert tumor-promoting effects [8]. The cellular concentration of p53 must be tightly regulated. While it can suppress tumors, high level of p53 may accelerate the aging process by excessive apoptosis. The major regulator of $\mathrm{p} 53$ is $\mathrm{Mdm} 2$, which can trigger the degradation of $\mathrm{p} 53$ by the ubiquitin system $[9,10]$.

\section{Role of p53 in Extrinsic Pathway of Apoptosis}

p53 can activate the extrinsic apoptotic pathway through the induction of genes encoding three transmembrane proteins: Tumor necrosis factor receptor-1 (TNFR1), Fas and death receptor-5 (DR5), which on activation transducer signals to further activate the caspases responsible for apoptosis [11]. p53 induces Fas mRNA expression by binding to elements found in the promoter and first intron of the Fas gene. This induction along with the induction of DR5 and TNFR1 by p53 occurs in response to DNA damage and in turn promotes cell death through caspase-8 [12-17].

Apaf-1: Apoptotic protease activating factor-1); Bid: $\mathrm{BH} 3$ interacting-domain death agonist; Bax: Bcl-2-associated X protein; $\mathrm{Cyt}$ c: Cytrochrome c; FADD: Fas associated death domain; Puma: p53upregulated modulator of apoptosis; t-Bid: Truncated BH3 interactingdomain death agonist; Bax: Bcl-2-associated X protein; TRAIL: TNF related apoptosis-inducing ligand [18].

\section{Role of p53 in Intrinsic Pathway of Apoptosis}

The intrinsic apoptotic pathway is dominated by the Bcl-2 family of proteins which comprises of anti-apoptotic proteins eg., Bcl-XL, Bcl-2, etc, and pro-apoptotic proteins eg., Bax, Noxa, Puma, Bid, etc., and are responsible for the release of cytochrome $c$ from the mitochondria. Balance of pro- and anti-apoptotic bcl-2 proteins decides the sensitivity of cells to apoptotic stimuli like excess of proapoptotic proteins renders cells sensitive to apoptosis; while excess of anti-apoptotic proteins render cells more resistant to apoptosis. When there is an irreparable DNA damage, p53 induces the expression of Bcl-2 proapoptotic family members: Bax, Puma and Noxa, resulting in shifting of the balance towards pro-apoptotic effects [19-21]. p53

${ }^{*}$ Corresponding author: Rashmi Kumariya, Animal Biochemistry Division, National Dairy Research Institute, Karnal, Haryana-132001, India, E-mail: rash4787@gmail.com

Received September 24, 2012; Accepted October 17, 2012; Published October 19,2012

Citation: Kumariya R, Barui AK, Singh S (2012) p53-Cells' Inbuilt Mechanism to Inhibit Cancer through Apoptosis. J Cancer Sci Ther 4: 368-370. doi:10.4172/19485956.1000169

Copyright: (c 2012 Ibrahim AM, et al. This is an open-access article distributed under the terms of the Creative Commons Attribution License, which permits unrestricted use, distribution, and reproduction in any medium, provided the original author and source are credited. 


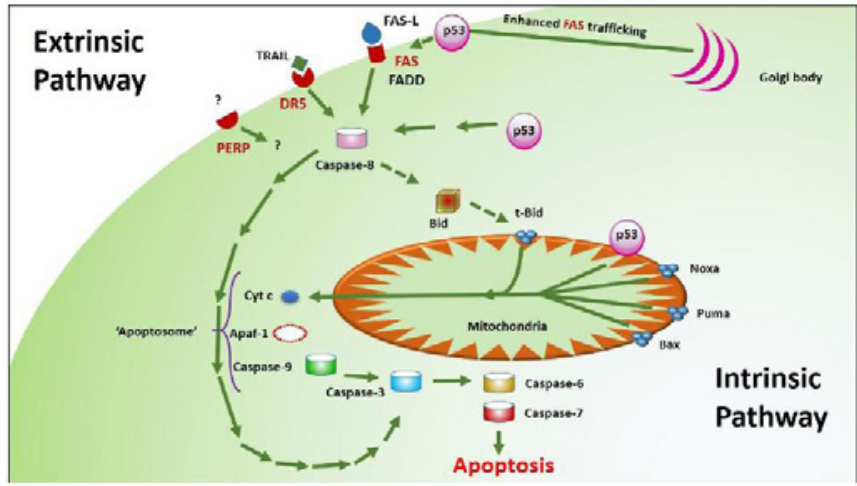

Figure 1: Schematic representation of pathways involved in p53 mediated apoptosis.

further induces the trimerization of the Bax, Puma and Noxa proteins in response to the DNA damage which may occur due to $\gamma$-irradiation, $\mathrm{X}$-ray irradiation, etc. resulting in the formation of the pores in mitochondria and the release of cytochrome-c and pro-apoptotic proteins from the intermembrane space. This release of cytochrome c from the mitochondria is a particularly important event in the induction of apoptosis as once it has been released into the cytosol, it is able to interact with a protein called Apaf-1 (Apoptotic protease activating factor-1), which leads to the recruitment of pro-caspase 9 into a multi-protein complex with cytochrome $\mathrm{c}$ and Apaf- 1 called the apoptosome [22]. Formation of the apoptosome leads to activation of caspase- 9 and the induction of apoptosis (Figure 1).

p53 also participates in apoptosis induction by acting directly at mitochondria. Localization of p53 to the mitochondria by using mitochondrial-import leader peptides occurs in response to apoptotic signals and precedes cytochrome $\mathrm{c}$ release and procaspase- 3 activation [23]. P53 also known to induce Bax and Bak oligomerization in the mitochondria, antagonizing the anti-apoptotic effect of $\mathrm{Bcl}-2$ and $\mathrm{Bcl}-$ $\mathrm{X}_{\mathrm{L}}$ and forms a complex with cyclophilin $\mathrm{D}$ in the mitochondrial inner membrane. These cause disruption of mitochondrial membranes and subsequent release of apoptogenic factors [24].

\section{Caspase Activation}

There are two categories of caspases: initiator caspases like, caspase-9, caspase-2, caspase- 8 and caspase-10 and effector caspases, caspase-3, caspase- 6 and caspase-7. Initiator caspases, caspase- 9 and caspase- 2 respond to changes in mitochondrial potential, whereas caspase- 8 and caspase- 10 sense activation of death receptors which cleave the pro-enzyme forms of the effector caspases, which further digest the essential targets that affect cell viability [11].

\section{Conclusion}

p53 family transcriptional targets have been identified as having the capacity to modulate various cellular processes including growth arrest, apoptosis, senescence, differentiation, and DNA repair. Deregulation of p53 has enormous influence on carcinogenesis as mutant p53 can induce an increased epigenetic instability of tumor cells, facilitating and accelerating the evolution of the tumor. Based on p53's critical role in carcinogenesis, multiple effective strategies for treating cancer can be developed by enhancing function of p 53 or increasing p 53 stability [25].

As p53 plays an important role in the cellular response to stress, it serves as a major barrier to carcinogenesis. This obstacle has to be removed in order for tumor development to proceed and restoration of $\mathrm{p} 53$ function is thus a potential key in anticancer therapies. Since $\mathrm{Mdm} 2$ is an important negative regulator of $\mathrm{p} 53, \mathrm{Mdm} 2$ hyperactivity may inhibit the function of p53 and lead to the development of a wide variety of cancers. Because the apoptotic function of p53 is critical for tumor suppression, induction of apoptotic pathways through p53-induced apoptotic targets may be an attractive strategy for anticancer treatment. Furthermore, the p53 apoptotic targets, unlike p53, are rarely mutated in human cancers. There is a clear consensus that restoration of the function of mutant p53 in tumor cells would also be of potential therapeutic benefit. This strategy should be specific to cancer cells, as normal cells contain no mutant p53. Understanding the mechanisms of p53's may ultimately provide novel targets and approaches to therapeutic manipulation of the p53 pathway in the treatment of cancer. Sound knowledge of p53 will help to develop highly effective strategies and novel drugs for cancer prevention and treatment with fewer side effects.

\section{References}

1. Kroemer G, Galluzzi L, Brenner C (2007) Mitochondrial membrane permeabilization in cell death. Physiol Rev 87: 99-163.

2. Fridman JS, Lowe SW (2003) Control of apoptosis by p53. Oncogene 22: 9030 9040

3. Kern SE, Kinzler KW, Bruskin A, Jarosz D, Friedman P, et al. (1991) Identification of $\mathrm{p} 53$ as a sequence-specific DNA-binding protein. Science 252 1708-1711.

4. Wei J, Zaika E, Zaika A (2012) p53 family: Role of protein isoforms in human cancer. J Nucleic Acids.

5. Selivanova G (2004) p53: fighting cancer. Curr Cancer Drug Targets 4: 385 402

6. Slee EA, O'Connor DJ, Lu X (2004) To die or not to die: how does p53 decide? Oncogene 23: 2809-2818.

7. Ozaki T, Nakagawara A (2011) Role of p53 in cell death and human cancers Cancers 3: 994-1013.

8. Suzuki K, Matsubara H (2011) Recent advances in p53 research and cancer treatment. J Biomed Biotechnol.

9. Yang Y, Li CC, Weissman AM (2004) Regulating the p53 system through ubiquitination. Oncogene 23: 2096-2106.

10. Goh AM, Coffill CR, Lane DP (2011) The role of mutant p53 in human cancer J Pathol 223: 116-126.

11. Maximov GK, Maximov KG (2008) The role of p53 tumor-suppressor protein in apoptosis and carcinogenesis. Biotechnol Biotec Eq 22: 664-668.

12. Chen G, Goeddel DV (2002) TNF-R1 signaling: a beautiful pathway. Science 296:1634-1635.

13. Liu X, Yue P, Khuri FR, Sun SY (2004) p53 upregulates death receptor 4 expression through an intronic p53 binding site. Cancer Res 64: 5078-5083.

14. O'Connor L, Harris AW, Strasser A (2000) CD95 (Fas/APO-1) and p53 signal apoptosis independently in diverse cell types. Cancer Res 60: 1217-1220.

15. Wajant $H(2002)$ The Fas signaling pathway: more than a paradigm. Science 296: 1634-1635.

16. Sheikh MS, Burns TF, Huang Y, Wu GS, Amundson S, et al. (1998) p53 dependent and -independent regulation of the death receptor KILLER/DR5 gene expression in response to genotoxic stress and tumor necrosis factor alpha. Cancer Res 58: 1593-1598.

17. Takimoto R, El-Deiry WS (2000) Wild-type p53 transactivates the KILLER/DR5 gene through an intronic sequence-specific DNA-binding site. Oncogene 19 1735-1743. 
Citation: Kumariya R, Barui AK, Singh S (2012) p53-Cells' Inbuilt Mechanism to Inhibit Cancer through Apoptosis. J Cancer Sci Ther 4: 368-370. doi:10.4172/1948-5956.1000169

18. Haupt S, Berger M, Goldberg Z, Haupt Y (2003) Apoptosis - the p53 network. J Cell Sci 116: 4077-4085.

19. Thornborrow EC, Patel S, Mastropietro AE, Schwartzfarb EM, Manfredi JJ (2002) A conserved intronic response element mediates direct p53-dependent transcriptional activation of both the human and murine bax genes. Oncogene 21: $990-999$

20. Nakano K, Vousden KH (2001) PUMA, a novel proapoptotic gene, is induced by p53. Mol Cell 7: 683-694.

21. Oda E, Ohki R, Murasawa H, Nemoto J, Shibue T, et al. (2000) Noxa, a $\mathrm{BH} 3$-only member of the $\mathrm{Bcl}-2$ family and candidate mediator of p53-induced apoptosis. Science 288: 1053-1058.
22. Cecconi F, Alvarez-Bolado G, Meyer BI, Roth KA, Gruss P (1998) Apaf1 (CED4 homolog) regulates programmed cell death in mammalian development. Cell 94: 727-737.

23. Matsuda K, Yoshida K, Taya Y, Nakamura K, Nakamura Y, et al. (2002) p53AIP1 regulates the mitochondrial apoptotic pathway. Cancer Res 62: 28832889.

24. Vaseva AV, Marchenko ND, Ji K, Tsirka SE, Holzmann S, et al. (2012) p53 Opens the Mitochondrial Permeability Transition Pore to Trigger Necrosis. Cell 149: $1536-1548$

25. George P (2011) p53 How crucial is its role in cancer? Int J Curr Pharm Res 3: $19-25$. 